\title{
Synthetic Methods
}

Abbreviations:

\begin{tabular}{|c|c|}
\hline $\mathrm{AcOH}$ & acetic acid \\
\hline $\mathrm{ACN}$ & acetonitrile \\
\hline app & apparent \\
\hline ATP & adenosine 5'-triphosphate \\
\hline BI-DIME & 3-(tert-butyl)-4-(2,6-dimethoxyphenyl)-2,3-dihydrobenzo[d][1,3]oxaphosphole \\
\hline BISPIN & 4,4,4',4',5,5,5',5'-octamethyl-2,2'-bi(1,3,2-dioxaborolane) \\
\hline BOC & tert-butyloxycarbonyl \\
\hline br & broad \\
\hline $\mathrm{Bu}$ & butyl \\
\hline CDI & carbonyldiimidazole \\
\hline d & doublet \\
\hline dd & doublet of doublets \\
\hline DCE & dichloroethane \\
\hline DCM & dichloromethane \\
\hline DIPEA & diisopropylethylamine \\
\hline DMA & dimethylacetamide \\
\hline DMAP & 4-dimethylaminopyridine \\
\hline DME & 1,4-dimethoxyethane \\
\hline DMF & $\mathrm{N}, \mathrm{N}$-dimethylformamide \\
\hline DMSO & dimethylsulfoxide \\
\hline ESI & electrospray ionization \\
\hline Et & ethyl \\
\hline EtOAc & ethyl acetate \\
\hline $\mathrm{h}$ & hour(s) \\
\hline HATU & $\begin{array}{l}\text { 1-[bis(dimethylamino)methylene]-1H-1,2,3-triazolo[4,5-b]pyridinium } \\
\text { hexafluorophosphate }\end{array}$ \\
\hline HBTU & $\begin{array}{l}\text { 1-[bis(dimethylamino)methylene]-1H-benzotriazoliumhexafluorophosphate(1-) 3- } \\
\text { oxide }\end{array}$ \\
\hline HOBt & 1-hydroxy-7-azabenzotriazole \\
\hline HPLC & high pressure liquid chromatography \\
\hline LCMS & liquid chromatography and mass spectrometry \\
\hline $\mathrm{MeCN}$ & acetonitrile \\
\hline $\mathrm{MeOH}$ & methanol \\
\hline $\mathrm{MHz}$ & mega hertz \\
\hline MS & mass spectrometry \\
\hline
\end{tabular}




\begin{tabular}{|c|c|}
\hline MW & microwave \\
\hline $\mathrm{m}$ & multiplet \\
\hline $\mathrm{mg}$ & milligram \\
\hline $\min$ & minutes \\
\hline $\mathrm{ml}$ & milliliter \\
\hline $\mathrm{mmol}$ & millimol \\
\hline $\mathrm{m} / \mathrm{z}$ & mass to charge ratio \\
\hline NBS & N-bromosuccinimide \\
\hline NMR & nuclear magnetic resonance \\
\hline $\mathrm{o} / \mathrm{n}$ & overnight \\
\hline $\mathrm{PdCl}_{2}(\mathrm{dppf})$ & 1,1'-Bis(diphenylphosphino)ferrocene-palladium(II)dichloride \\
\hline $\mathrm{Pd}(\mathrm{OAc})_{2}$ & palladium(II) acetate \\
\hline $\mathrm{Pd} / \mathrm{C}$ & palladium on carbon \\
\hline $\mathrm{Ph}$ & phenyl \\
\hline ppm & parts per million \\
\hline rac & racemic \\
\hline RBF & round bottom flask \\
\hline $\mathrm{Rt}$ & retention time \\
\hline RT & room temperature \\
\hline s & singlet \\
\hline sat. & saturated \\
\hline SCX & strong cation exchange sorbent column \\
\hline SM & starting material \\
\hline $\mathrm{t}$ & triplet \\
\hline TBME & methyl tert-butyl ether \\
\hline $\mathrm{tBu}$ & tertiary butyl \\
\hline TEA & triethylamine \\
\hline tert & tertiary \\
\hline TFA & trifluoroacetic acid \\
\hline THF & tetrahydrofuran \\
\hline TLC & thin layer chromatography \\
\hline
\end{tabular}




\section{Purification}

Purification of intermediates and final products was carried out via either normal or reverse phase chromatography. Normal phase chromatography was carried out using prepacked $\mathrm{SiO}_{2}$ cartridges (e.g., RediSep $®$ Rf columns from Teledyne Isco, Inc.) eluting with gradients of appropriate solvent systems (heptane and ethyl acetate, or $\mathrm{DCM}$ and $\mathrm{MeOH}$, unless otherwise indicated). Reverse phase preparative HPLC was carried out using the methods described below:

(1) Basic method: XBridge $5 \mu \mathrm{m}$ column, $5 \mathrm{mM} \mathrm{NH}_{4} \mathrm{OH}$ in acetonitrile and water.

(2) TFA method: Sunfire $5 \mu \mathrm{m}$ column, $0.1 \%$ TFA in acetonitrile and water.

(3) Formic acid method: XBridge $5 \mu \mathrm{m}$ column; $0.1 \%$ formic acid in acetonitrile and water.

All of the above three HPLC methods run a focused gradient from the starting $\%$ acetonitrile to the final $\%$ acetonitrile. The initial and final conditions for each gradient are as follows: Method 0: $2-12 \%$ acetonitrile; Method 1: $7.5-20 \%$ acetonitrile; Method 2: 10-30\% acetonitrile; Method 3: 15-40\% acetonitrile; Method 4: 25-50\% acetonitrile; Method 5: 35-60\% acetonitrile; Method 6: 45-70\% acetonitrile; Method 7: 55-80\% acetonitrile; Method 8: 65-95\% acetonitrile; and Method 9: 5-95\% acetonitrile. 


\section{General Synthetic Schemes}

\section{General Procedure for Methylation}<smiles>C[C@H](OC(C)(C)C)C(C)(C)C</smiles>

To a stirring solution of cesium carbonate $(4.04 \mathrm{~g}, 12.39 \mathrm{mmol})$ in THF (Volume: $41.3 \mathrm{ml})$ was added 4bromo-7-fluoroisoquinolinone $(1 \mathrm{~g}, 4.13 \mathrm{mmol})$. The reaction was sonicated vigorously, after which time iodomethane $(0.310 \mathrm{ml}, 4.96 \mathrm{mmol})$ was added dropwise and the reaction was allowed to stir at RT o/n.

The following morning, the reaction was concentrated by rotary evaporation to remove THF. The crude material was diluted with water and extracted with EtOAc. The organics were washed $3 x$ with water, then brine, then filtered over a bed of magnesium sulfate and concentrated to afford the product as an offyellow solid (950 mg, $3.71 \mathrm{mmol}, 90 \%$ yield).

\section{General Procedure for Bromination}<smiles>Cn1ccc2ccc(C#N)cc2c1=O</smiles>

NBS (276 mg, $1.553 \mathrm{mmol})$ and isoquinolinone $(260 \mathrm{mg}, 1.412 \mathrm{mmol})$ were suspended in acetonitrile $(0.1$ $M)$ in a $50 \mathrm{~mL} R B F$ equipped with a stir bar and stirred at RT o/n.

The following morning, the reaction was concentrated to a solid, re-suspended in EtOAc and filtered to remove the succinimide byproduct. The organic layer was concentrated to afford the desired product as a cream solid (268 mg, $1.019 \mathrm{mmol}, 72.2 \%$ yield).

\section{General Procedure for Borylation}<smiles>Cn1cc(Br)c2ccc(F)cc2c1=O</smiles>

KOAc, BISPIN, $\mathrm{Pd}(\mathrm{dppf}) \mathrm{Cl}_{2}$<smiles>Cn1cc(B2OC(C)(C)C(C)(C)O2)c2ccc(F)cc2c1=O</smiles>

Isoquinolinone (500 mg, $1.93 \mathrm{mmol})$, BISPIN $(744 \mathrm{mg}, 2.93 \mathrm{mmol})$, potassium acetate $(479 \mathrm{mg}, 4.88$ $\mathrm{mmol})$ and $\mathrm{Pd}(\mathrm{dppf}) \mathrm{Cl}_{2}(286 \mathrm{mg}, 0.391 \mathrm{mmol})$ were suspended in dioxane $(0.1 \mathrm{M})$ in a $20 \mathrm{~mL}$ pressure relief vial under $\mathrm{N}_{2}$. The mixture (a brown-orange suspension) was stirred at $90^{\circ} \mathrm{C}$ (suspension became 
darker in color with heat) o/n.

The following morning, LC reveals conversion to the desired borane species. The reaction was diluted with DCM and washed $3 x$ with sat. sodium bicarbonate. The organic layers were combined, passed through a bed of sodium sulfate, and concentrated to a brown oil. Assume quantitative yield, this material was used directly without further manipulation.

\section{General Procedure for Cross-Coupling - ROUTE A}<smiles>COc1cc(C=O)cc(OC)c1B(O)O</smiles><smiles>Cn1cc(Br)c2ccncc2c1=O</smiles>

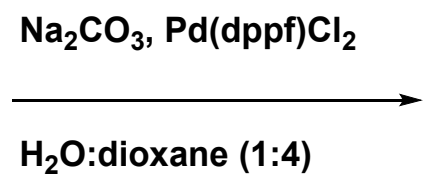<smiles>COc1cc(C=O)cc(OC)c1-c1cn(C)c(=O)c2cnccc12</smiles>

$\mathrm{Pd}(\mathrm{dppf}) \mathrm{Cl}_{2}$ (30.6 mg, $0.042 \mathrm{mmol}$ ), sodium carbonate $(66.5 \mathrm{mg}, 0.627 \mathrm{mmol}$ ), napthyridinone (50 $\mathrm{mg}$, $0.209 \mathrm{mmol})$ and 4-formyl-2,6-dimethoxy phenylboronic acid $(43.9 \mathrm{mg}, 0.209 \mathrm{mmol})$ were suspended in 4:1 dioxane/water $(0.1 \mathrm{M})$ in a $2 \mathrm{~mL} \mathrm{MW}$ vial. The reaction was sealed and heated in a $\mathrm{MW}$ at $160{ }^{\circ} \mathrm{C}$ for 5 min.

The reaction was concentrated to a solid, suspended in a small volume $(1 \mathrm{~mL})$ of 1:1 ACN/water and filtered. The crude material was purified by HPLC (basic, method 2, Rt 4.45) to afford the desired product as a brown solid (18 $\mathrm{mg}, 0.055 \mathrm{mmol}, 26.5 \%$ yield).

\section{General Procedure for Cross-Coupling - ROUTE B}<smiles>COc1cc(CN(C)C)cc(OC)c1Br</smiles><smiles>Cn1cc(B2OC(C)(C)C(C)(C)O2)c2ccc(F)cc2c1=O</smiles>

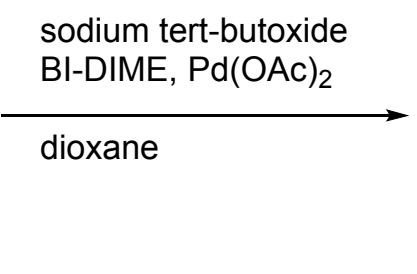<smiles>COc1cc(CN(C)C)cc(OC)c1-c1cn(C)c(=O)c2cc(F)ccc12</smiles>

$\mathrm{Pd}(\mathrm{OAc})_{2}(2.457 \mathrm{mg}, 10.94 \mu \mathrm{mol})$, sodium tert-butoxide (31.5 mg, $\left.0.328 \mathrm{mmol}\right)$, BI-DIME ligand (7.23 mg, $0.022 \mathrm{mmol})$, isoquinolinone $(33.2 \mathrm{mg}, 0.109 \mathrm{mmol}$ ) and bromo dimethylamine $(30 \mathrm{mg}, 0.109 \mathrm{mmol})$ were suspended in dioxane $(0.1 \mathrm{M})$ in a $2 \mathrm{~mL} \mathrm{MW}$ vial. The reaction was sealed and heated in a $\mathrm{MW}$ at $160^{\circ} \mathrm{C}$ for $1 \mathrm{hr}$.

The reaction was quenched by the addition of sodium bicarbonate $(2 \times 5 \mathrm{~mL})$ and the crude material was extracted 3 times with EtOAc. The combined organic layers were dried over $\mathrm{MgSO}_{4}$, filtered, and 
concentrated to a brown oil. The crude material was purified by HPLC (acidic, method 2, Rt 2.55) to afford the desired product as a white solid $(6.7 \mathrm{mg}, 0.017 \mathrm{mmol}, 15.7 \%$ yield $)$

\section{General Procedure for Reductive Aminations}<smiles>COc1cc(C=O)cc(OC)c1-c1cn(C)c(=O)c2cnccc12</smiles>
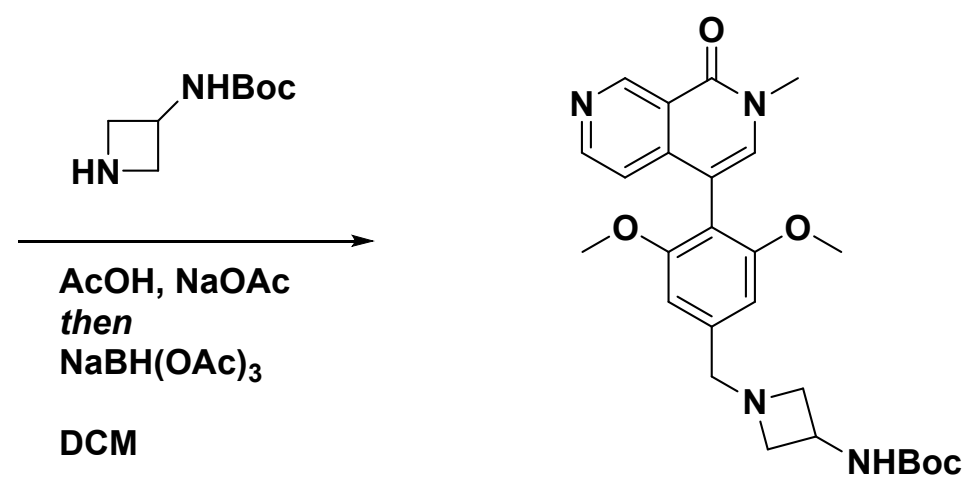

In a $4 \mathrm{~mL}$ pressure relief vial, a mixture of sodium acetate $(6.37 \mathrm{mg}, 0.078 \mathrm{mmol})$, acetic acid $(3.18 \mu \mathrm{l}$, $0.055 \mathrm{mmol}$ ), 3-N-Boc-amino azetidine $(13.38 \mathrm{mg}, 0.078 \mathrm{mmol})$, and aldehyde $(18 \mathrm{mg}, 0.055$ $\mathrm{mmol})$ in DCM $(0.1 \mathrm{M})$ were stirred at $0^{\circ} \mathrm{C}$ for 30 minutes under a stream of nitrogen gas. Then, sodium triacetoxyborohydride $(23.52 \mathrm{mg}, 0.111 \mathrm{mmol})$ was added in one portion and the reaction mixture was stirred at RT o/n.

The following morning, saturated $\mathrm{NaHCO}_{3}$ solution was added and the layers were separated. The aqueous layer was extracted $3 x$ with $\mathrm{DCM}$. The combined organic layers were dried with $\mathrm{Na}_{2} \mathrm{SO}_{4}$ and concentrated onto a bed of celite. The material was purified by ISCO (0-10\% MeOH in DCM) to afford the target compound as a light brown solid ( $14 \mathrm{mg}, 0.029 \mathrm{mmol}, 52.5 \%$ yield).

\section{General Procedure for BOC-deprotection}<smiles>COc1cc(CN2CC(NC(=O)OC(C)(C)C)C2)cc(OC)c1-c1cn(C)c(=O)c2cnccc12</smiles><smiles>COc1cc(CN2CC(N)C2)cc(OC)c1-c1cn(C)c(=O)c2cnccc12</smiles>

To a stirring solution of BOC-protected amine (14 $\mathrm{mg}, 0.029 \mathrm{mmol})$ in DCM $(0.1 \mathrm{M})$ at RT was added TFA $(33.7 \mu \mathrm{l}, 0.437 \mathrm{mmol})$. The reaction was allowed to stir at RT o/n.

The following morning, the crude reaction was concentrated to an oil, re-suspended in $1 \mathrm{~mL}$ of $1: 1$ ACN:water and was purified by HPLC (basic, method 2, Rt 3.12). The product fractions were combined and concentrated to afford the target as a white solid $(7.3 \mathrm{mg}, 0.019 \mathrm{mmol}, 65.2 \%$ yield). 
General Procedure for Amide Couplings

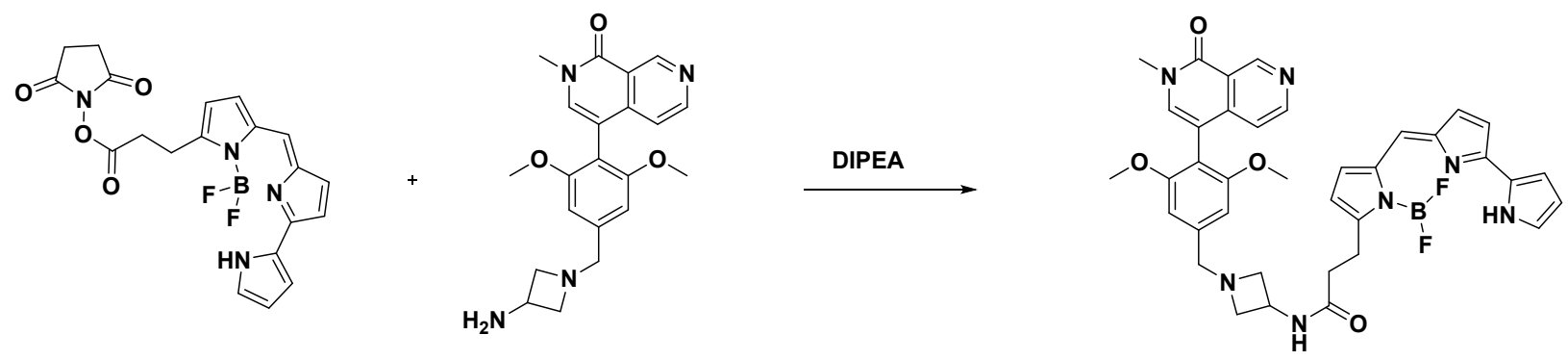

To a solution of azetidine amine $(10 \mathrm{mg}, 0.026 \mathrm{mmol})$ in DCM $(0.1 \mathrm{M})$ at RT was added DIPEA (8.17 $\mu$, $0.047 \mathrm{mmol}$ ) and stirred for five minutes. Then BODIPY dye ( $5 \mathrm{mg}, 0.012 \mathrm{mmol})$ was added and the reaction stirred for $2.5 \mathrm{hr}$.

Upon complete consumption of SM by LCMS, the crude material was concentrated to a solid, resuspended in ACN, and purified by HPLC (basic, method 4, Rt 3.80). The product fractions were combined and concentrated to afford the target compound as a purple solid $(1.9 \mathrm{mg}, 0.00261 \mathrm{mmol}$, $22.3 \%$ yield).

General Procedure for Saponification<smiles>COC(=O)c1ccc2c(Br)cn(C)c(=O)c2c1</smiles><smiles>O=[W]O[Mg]</smiles><smiles>Cn1cc(Br)c2ccc(C(=O)O)cc2c1=O</smiles>

To the methyl ester $(150 \mathrm{mg}, 0.507 \mathrm{mmol})$ in $3: 1$ solution of THF/water $(0.1 \mathrm{M})$ vortexing at RT was added $\mathrm{LiOH}$ dropwise (507 uL, $1.013 \mathrm{mmol}$ ). The reaction stirred overnight.

The following morning, LC revealed consumption of SM to the desired product. The crude material was concentrated to a solid and used directly on the subsequent steps, assuming quantitative yield of the lithium salt.

\section{General Procedure for HATU-mediated Amidation}<smiles>Cn1cc(Br)c2ccc(C(=O)O)cc2c1=O</smiles>

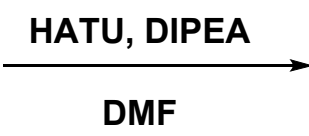<smiles>CNC(=O)c1ccc2c(Br)cn(C)c(=O)c2c1</smiles>

To a solution of carboxylic acid (143 mg, $0.507 \mathrm{mmol})$ in DMF $(0.25 \mathrm{M})$ at RT was added DIPEA (328 mg, $2.54 \mathrm{mmol})$, and amine $(68 \mathrm{mg}, 1.014 \mathrm{mmol})$ and the reaction stirred for five minutes. Then HATU (231 $\mathrm{mg}, 0.608 \mathrm{mmol}$ ) was added and the reaction stirred for an additional $2.5 \mathrm{hr}$.

When LCMS revealed complete consumption of the acid, the crude material was concentrated onto a bed 
of celite and purified by ISCO $(0-10 \% \mathrm{MeOH}$ in DCM). The desired fractions were combined and concentrated to afford the target compound as a brown solid $(27.6 \mathrm{mg}, 0.093 \mathrm{mmol}, 18.3 \%$ yield).

\section{Compound Synthesis and Characterization}<smiles>COc1cc(-c2cn(C)c(=O)c3cnccc23)c(OC)cc1CN1CC(N)C1</smiles>

4-(4-((3-aminoazetidin-1-yl)methyl)-2,5-dimethoxyphenyl)-2-methyl-2,7-naphthyridin-1(2H)-one $59.9 \%, 0.071 \mathrm{mmol}, 27.3 \mathrm{mg}$

\section{Compound 1}

1H NMR (400 MHz, Methylene Chloride-d2) $\delta 9.59$ (d, J = 0.9 Hz, 1H), 8.64 (d, J = 5.6 Hz, 1H), 7.29 (s, 1H), 7.09 (dd, J = 4.5, $1.0 \mathrm{~Hz}, 2 \mathrm{H}), 6.78(\mathrm{~s}, 1 \mathrm{H}), 3.82(\mathrm{~s}, 3 \mathrm{H}), 3.72(\mathrm{~s}, 3 \mathrm{H}), 3.65(\mathrm{~s}, 4 \mathrm{H}), 2.84$ (t, J = 6.9 $\mathrm{Hz}, 2 \mathrm{H}), 1.73(\mathrm{~s}, 4 \mathrm{H})$.

$[\mathrm{M}+\mathrm{H}]=381.0, \mathrm{Rt}=0.64 \mathrm{~min}$ 


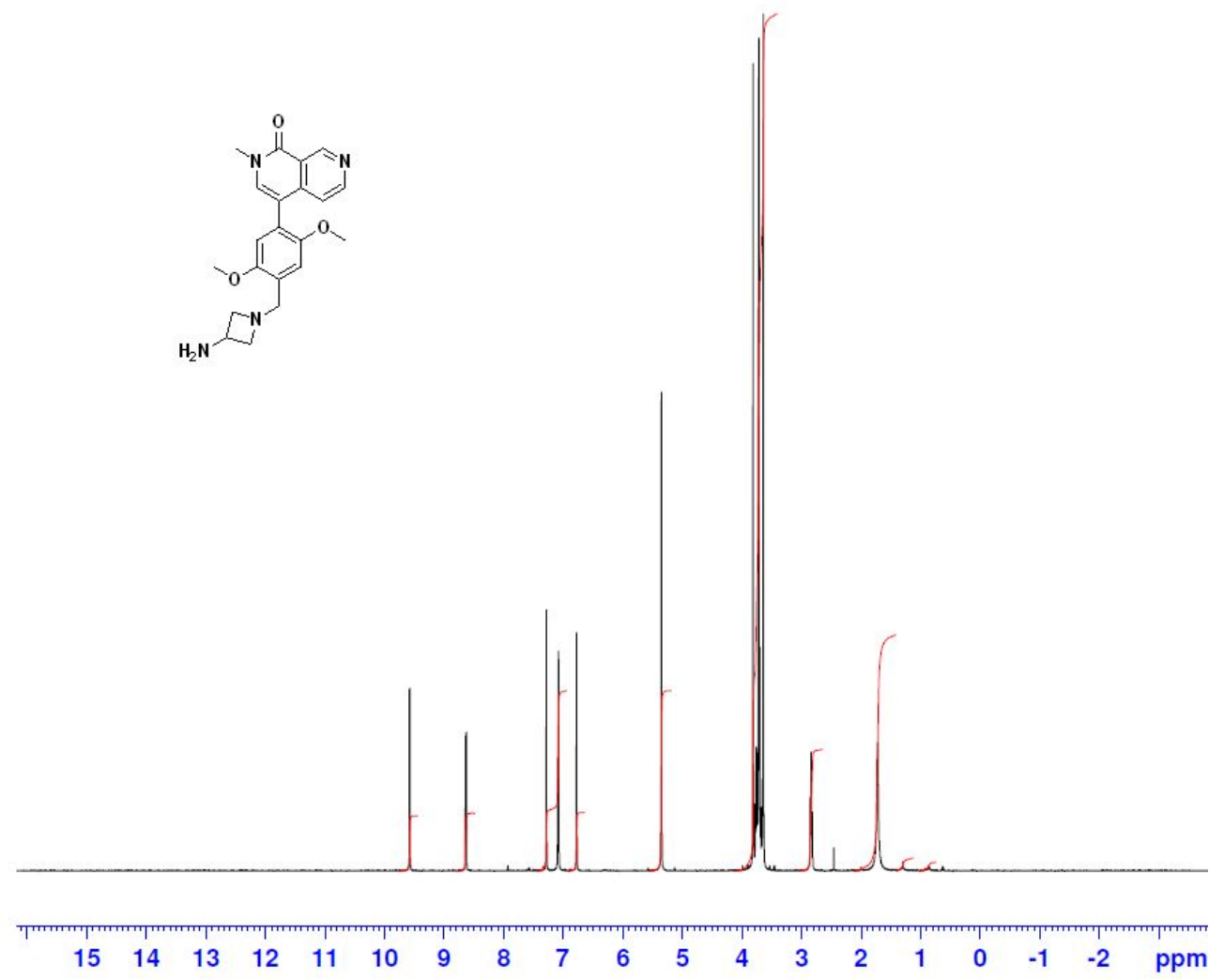<smiles>COc1cc(-c2cn(C)c(=O)c3cnccc23)c(OC)cc1CN1CC(NC(=O)OC(C)(C)C)C1</smiles>

tert-butyl (1-(2,5-dimethoxy-4-(2-methyl-1-oxo-1,2-dihydro-2,7-naphthyridin-4-yl)benzyl)azetidin-3yl)carbamate

Intermediate 1D

1H NMR (400 MHz, CHLOROFORM-d) $\delta$ ppm 1.46 (s, 9 H) $2.76-4.03(\mathrm{~m}, 17 \mathrm{H}) 6.74(\mathrm{~s}, 1 \mathrm{H}) 6.99-7.08$ $(\mathrm{m}, 2 \mathrm{H}) 7.24(\mathrm{~s}, 1 \mathrm{H}) 8.65(\mathrm{~d}, \mathrm{~J}=5.81 \mathrm{~Hz}, 1 \mathrm{H}) 9.68(\mathrm{~d}, \mathrm{~J}=0.76 \mathrm{~Hz}, 1 \mathrm{H})$

$[\mathrm{M}+\mathrm{H}]: 481.4, \mathrm{Rt}=0.90 \mathrm{~min}$ 
<smiles>COc1cc(-c2cn(C)c(=O)c3cnccc23)c(OC)cc1C=O</smiles>

2,5-dimethoxy-4-(2-methyl-1-oxo-1,2-dihydro-2,7-naphthyridin-4-yl)benzaldehyde

Intermediate 1C

1H NMR (400 MHz, CHLOROFORM-d) $\delta$ ppm 3.69 (s, 3 H) 3.76 (s, 3 H) 3.94 (s, 3 H) 6.93 (s, 1 H) 7.04 (d, J=5.56 Hz, 1 H) 7.29 (s, 1 H) $7.48(\mathrm{~s}, 1 \mathrm{H}) 8.67$ (d, J=5.56 Hz, 1 H) $9.70(\mathrm{~s}, 1 \mathrm{H}) 10.52(\mathrm{~s}, 1 \mathrm{H})$

$[\mathrm{M}+\mathrm{H}]: 325.1, \mathrm{Rt}=0.73 \mathrm{~min}$

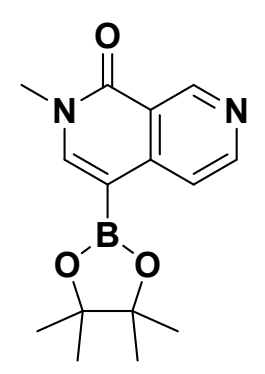

2-methyl-4-(4,4,5,5-tetramethyl-1,3,2-dioxaborolan-2-yl)-2,7-naphthyridin-1(2H)-one

Intermediate 1B

$[\mathrm{M}+\mathrm{H}]: 287.0, \mathrm{Rt}=0.73 \mathrm{~min}$<smiles>COc1cc(C=O)c(OC)cc1Br</smiles>

Commercially available (CAS 31558-40-4); 4-Bromo-3,5-dimethoxybenzaldehyde 
<smiles>Cn1cc(Br)c2ccncc2c1=O</smiles>

4-bromo-2-methyl-2,7-naphthyridin-1(2H)-one

Intermediate 1A

1H NMR (400 MHz, CHLOROFORM-d) $\delta$ ppm 3.64 (s, 3 H) 7.57 (s, 1 H) 7.62 (d, J=5.56 Hz, 1 H) 8.87 (d, $\mathrm{J}=5.56 \mathrm{~Hz}, 1 \mathrm{H}) 9.62(\mathrm{~s}, 1 \mathrm{H})$

$[\mathrm{M}+\mathrm{H}]: 239.1, \mathrm{Rt}=0.54 \mathrm{~min}$<smiles>O=c1[nH]cc(Br)c2ccncc12</smiles>

Commercially available (CAS 3951-95-9); 4-Bromo-1(2H)-isoquinoline<smiles>COc1ccc2ccccc2c1-c1cn(C)c(=O)c2cnccc12</smiles>

4-(2-methoxynaphthalen-1-yl)-2-methyl-2,7-naphthyridin-1(2H)-one $43.0 \%, 0.043 \mathrm{mmol}, 13.6 \mathrm{mg}$

\section{Compound 2}

${ }^{1} \mathrm{H}$ NMR (400 MHz, Chloroform-d) $\delta 9.64(\mathrm{~s}, 1 \mathrm{H}), 8.44(\mathrm{~d}, J=5.7 \mathrm{~Hz}, 1 \mathrm{H}), 7.93(\mathrm{~d}, J=9.0 \mathrm{~Hz}, 1 \mathrm{H}), 7.86-$ $7.77(\mathrm{~m}, 1 \mathrm{H}), 7.39-7.27(\mathrm{~m}, 4 \mathrm{H}), 7.21(\mathrm{~s}, 1 \mathrm{H}), 6.75(\mathrm{~d}, J=5.6 \mathrm{~Hz}, 1 \mathrm{H}), 3.76(\mathrm{~s}, 3 \mathrm{H}), 3.62(\mathrm{~s}, 3 \mathrm{H})$.

$[\mathrm{M}+\mathrm{H}]=317.2, \mathrm{Rt}=0.90 \mathrm{~min}$ 

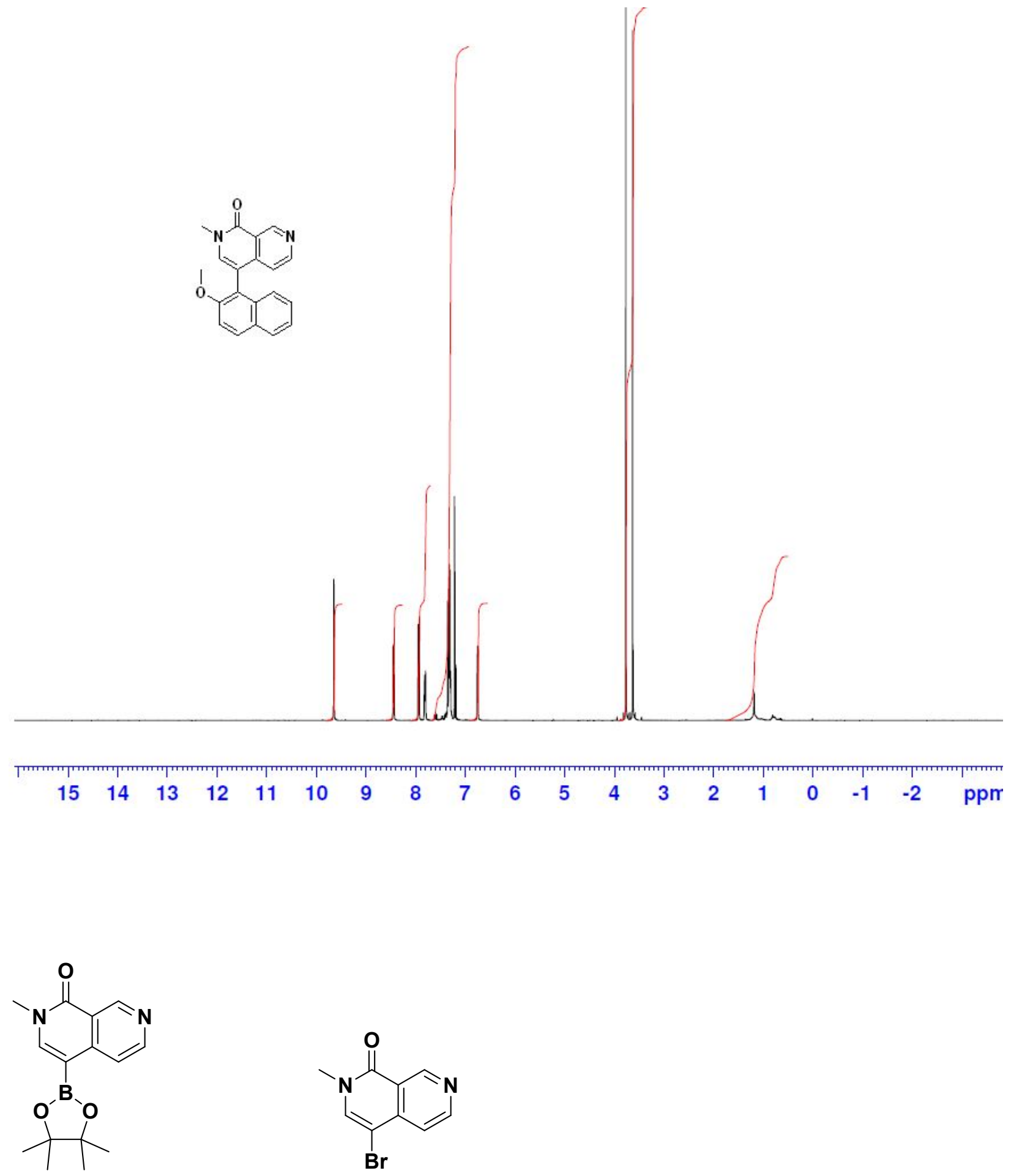

Intermediates $1 \mathrm{~A}+1 \mathrm{~B}$

See above 
<smiles>O=c1[nH]cc(Br)c2ccncc12</smiles>

Commercially available (CAS 3951-95-9); 4-Bromo-1(2H)-isoquinoline<smiles>COc1ccc2ccccc2c1Br</smiles>

Commercially available (CAS 3401-47-6); 1-Bromo-2-methoxynapthalene<smiles>COc1cc(CN(C)C)cc(OC)c1-c1cn(C)c(=O)c2cnccc12</smiles>

4-(4-((dimethylamino)methyl)-2,6-dimethoxyphenyl)-2-methyl-2,7-naphthyridin-1(2H)-one $43.0 \%, 0.043 \mathrm{mmol}, 13.6 \mathrm{mg}$

\section{Compound 3}

${ }^{1} \mathrm{H}$ NMR $(400 \mathrm{MHz}$, Chloroform-d) $\delta 9.59(\mathrm{~d}, J=0.9 \mathrm{~Hz}, 1 \mathrm{H}), 8.51(\mathrm{~d}, J=5.6 \mathrm{~Hz}, 1 \mathrm{H}), 7.09(\mathrm{~s}, 1 \mathrm{H}), 6.81$ $(\mathrm{d}, J=5.6 \mathrm{~Hz}, 1 \mathrm{H}), 6.70(\mathrm{~s}, 2 \mathrm{H}), 3.75-3.47(\mathrm{~m}, 11 \mathrm{H}), 2.47(\mathrm{~d}, J=60.0 \mathrm{~Hz}, 6 \mathrm{H})$.

$[\mathrm{M}+\mathrm{H}]=354.2, \mathrm{Rt}=0.75 \mathrm{~min}$ 

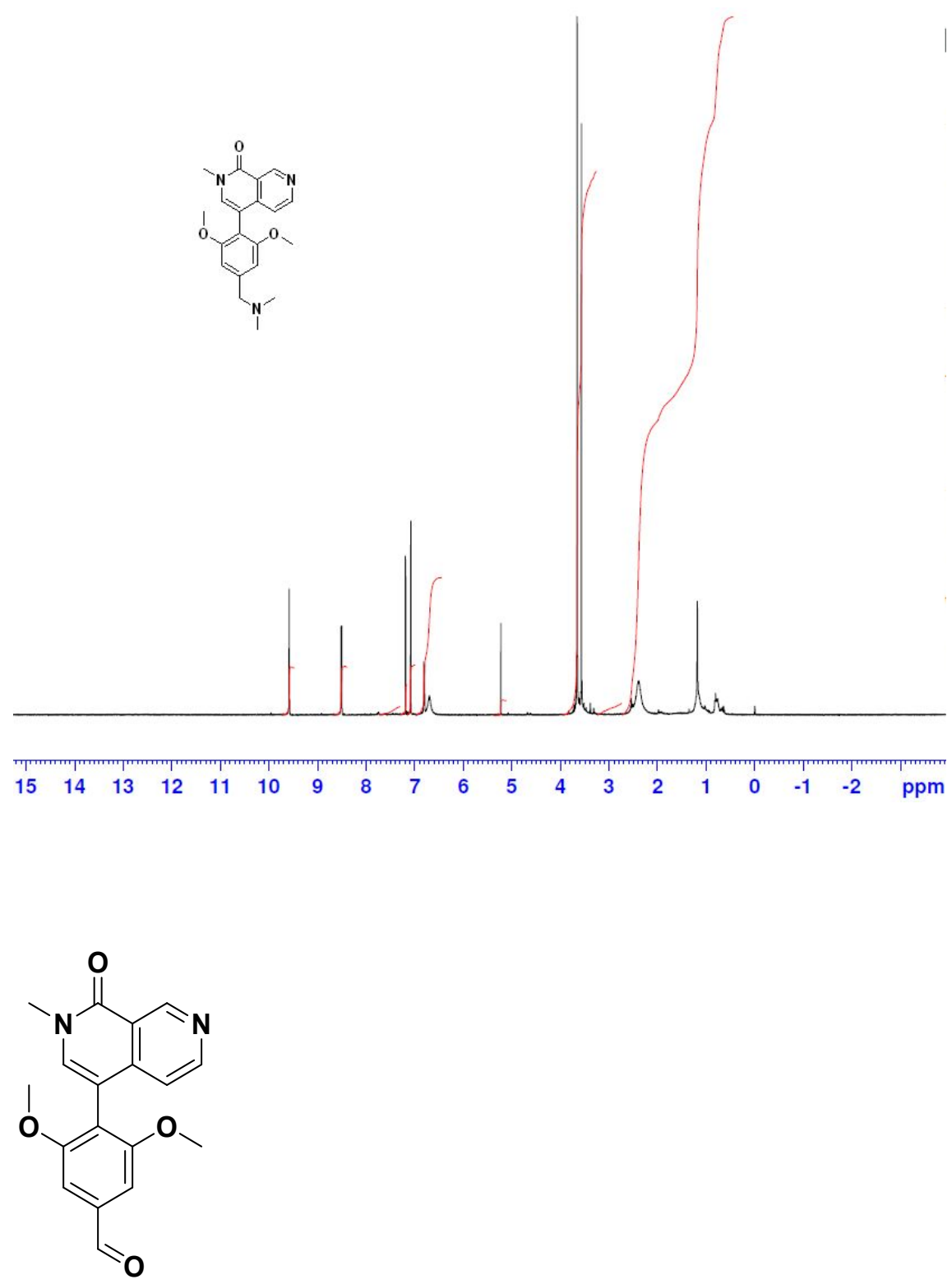

Intermediate 3A

3,5-dimethoxy-4-(2-methyl-1-oxo-1,2-dihydro-2,7-naphthyridin-4-yl)benzaldehyde $[\mathrm{M}+\mathrm{H}]: 325.0, \mathrm{Rt}=0.58 \mathrm{~min}$

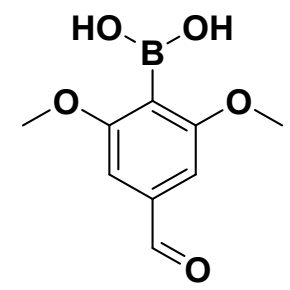

Commercially available (CAS 1256355-34-6); 2,6-Dimethoxy-4-forymlphenylboronic acid 
<smiles>COc1cc(-c2cn(C)c(=O)c3cncnc23)c(OC)cc1CN(C)C</smiles>

8-(4-((dimethylamino)methyl)-2,5-dimethoxyphenyl)-6-methylpyrido[4,3-d]pyrimidin-5(6H)-one $11.5 \%, 0.011 \mathrm{mmol}, 3.3 \mathrm{mg}$

\section{Compound 4}

${ }^{1} \mathrm{H}$ NMR $(400 \mathrm{MHz}$, Chloroform-d) $\delta 9.74(\mathrm{~d}, J=1.2 \mathrm{~Hz}, 1 \mathrm{H}), 9.33(\mathrm{~d}, J=9.9 \mathrm{~Hz}, 1 \mathrm{H}), 8.44(\mathrm{~s}, 1 \mathrm{H}), 7.62$ $(\mathrm{d}, J=10.4 \mathrm{~Hz}, 1 \mathrm{H}), 6.96(\mathrm{~d}, J=13.0 \mathrm{~Hz}, 1 \mathrm{H}), 4.25(\mathrm{~s}, 2 \mathrm{H}), 3.87(\mathrm{~d}, J=9.8 \mathrm{~Hz}, 3 \mathrm{H}), 3.78(\mathrm{~d}, J=12.5 \mathrm{~Hz}$, $3 \mathrm{H}), 3.70(\mathrm{~d}, J=2.6 \mathrm{~Hz}, 3 \mathrm{H}), 2.74(\mathrm{~d}, J=45.3 \mathrm{~Hz}, 6 \mathrm{H})$.

$[\mathrm{M}+\mathrm{H}]=355.2, \mathrm{Rt}=0.63 \mathrm{~min}$

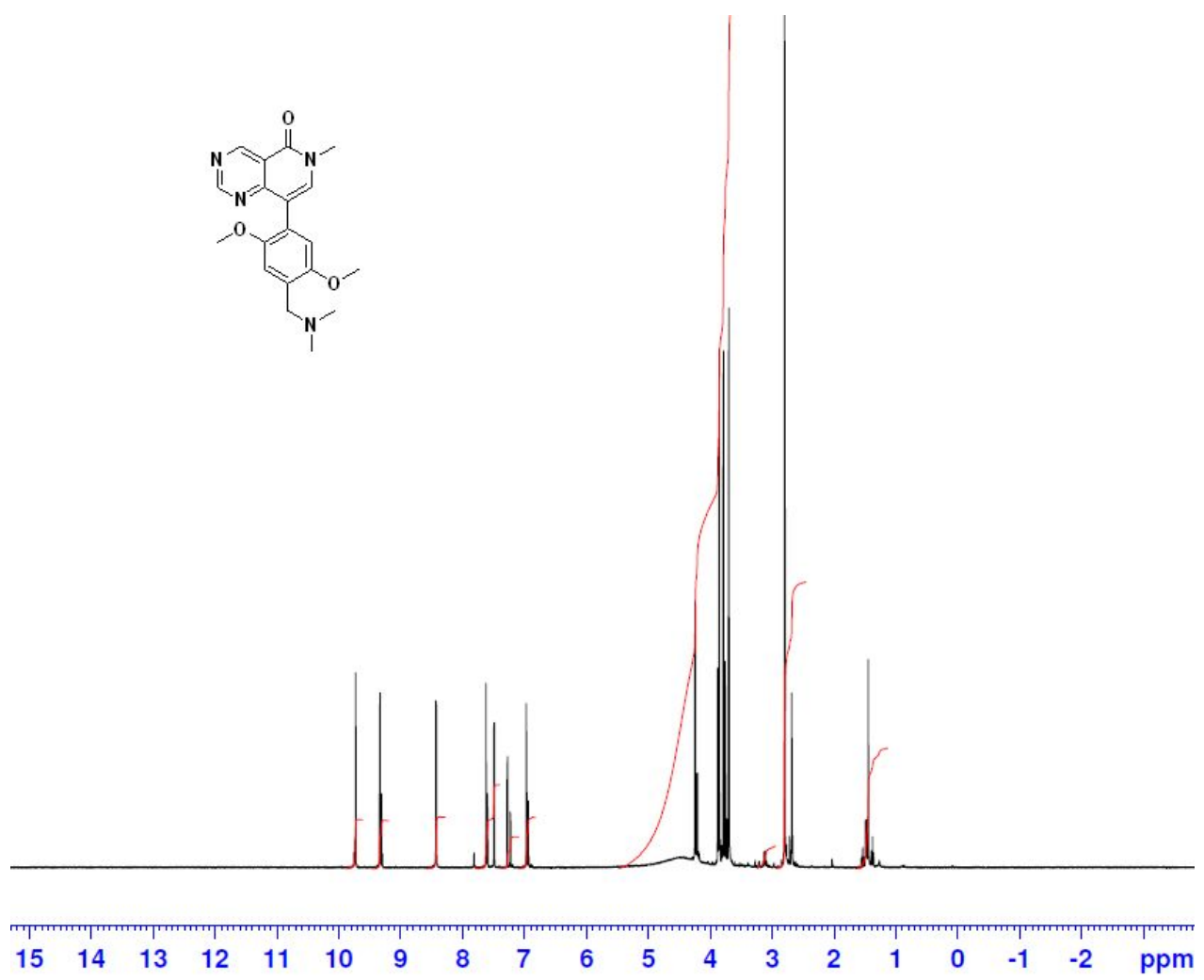

See patent (WO2016/139361 A1; EP3265453 A1; US2018/44335 A1; JP2018/507238 A) for intermediate analysis. 
<smiles>COc1cc(-c2cn(C)c(=O)c3cc([N+](=O)[O-])ccc23)c(OC)cc1CN(C)C</smiles>

4-(4-((dimethylamino)methyl)-2,5-dimethoxyphenyl)-2-methyl-7-nitroisoquinolin-1(2H)-one $11.5 \%, 0.011 \mathrm{mmol}, 3.3 \mathrm{mg}$

\section{Compound 5}

${ }^{1} \mathrm{H}$ NMR $(400 \mathrm{MHz}$, Chloroform-d) $\delta 9.74(\mathrm{~d}, J=1.2 \mathrm{~Hz}, 1 \mathrm{H}), 9.33(\mathrm{~d}, J=9.9 \mathrm{~Hz}, 1 \mathrm{H}), 8.44(\mathrm{~s}, 1 \mathrm{H}), 7.62$ $(\mathrm{d}, J=10.4 \mathrm{~Hz}, 1 \mathrm{H}), 6.96(\mathrm{~d}, J=13.0 \mathrm{~Hz}, 1 \mathrm{H}), 4.25(\mathrm{~s}, 2 \mathrm{H}), 3.87(\mathrm{~d}, J=9.8 \mathrm{~Hz}, 3 \mathrm{H}), 3.78(\mathrm{~d}, J=12.5 \mathrm{~Hz}$, $3 \mathrm{H}), 3.70(\mathrm{~d}, J=2.6 \mathrm{~Hz}, 3 \mathrm{H}), 2.74(\mathrm{~d}, J=45.3 \mathrm{~Hz}, 6 \mathrm{H})$.

$[\mathrm{M}+\mathrm{H}]=398.2, \mathrm{Rt}=0.98 \mathrm{~min}$
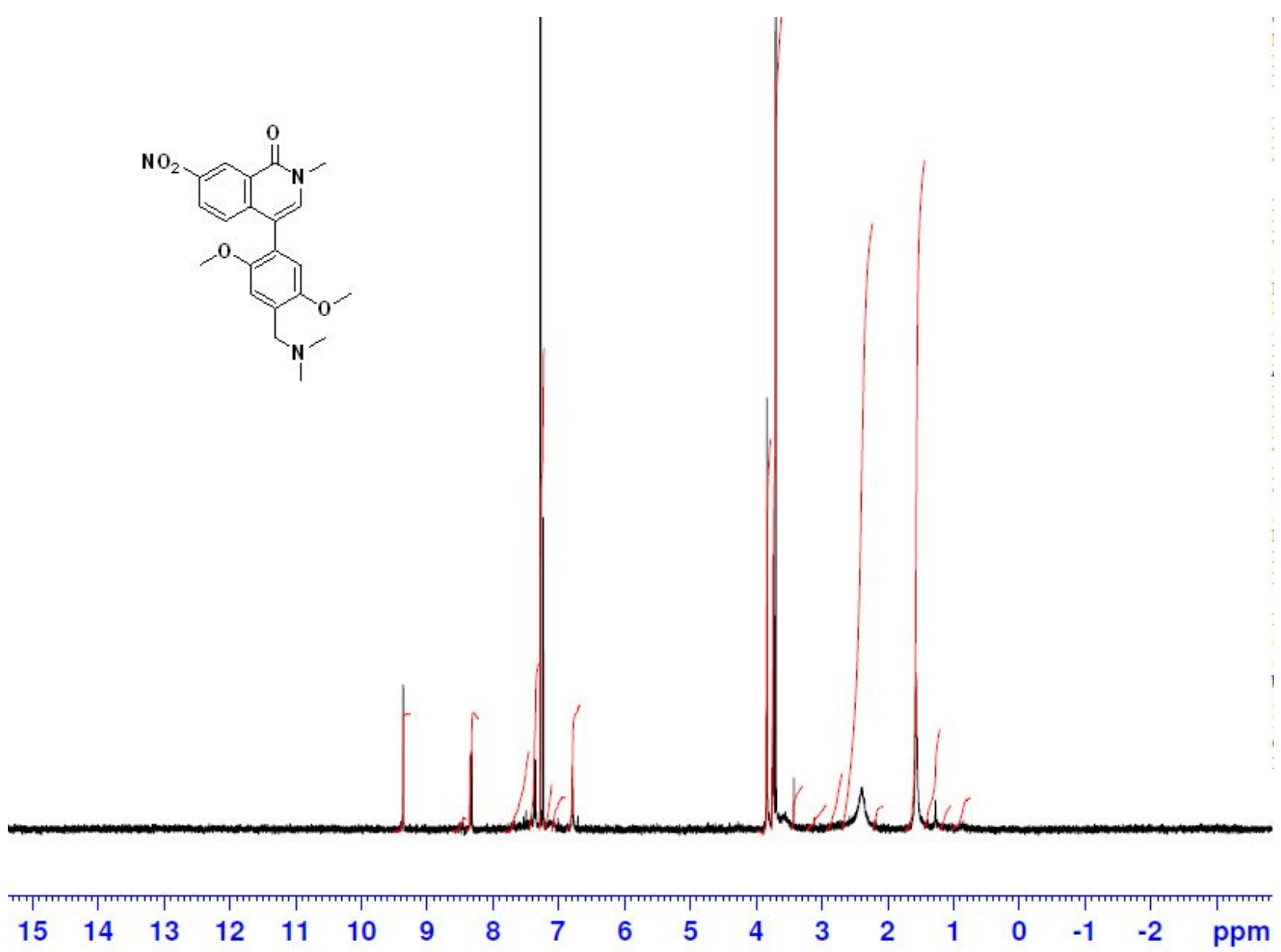
<smiles>O=c1[nH]cc(Br)c2ccc([N+](=O)[O-])cc12</smiles>

Commercially available (CAS 1036390-36-9); 4-Bromo-7-nitroisoquinolin-1(2H)-one<smiles>COc1cc(B2OC(C)(C)C(C)(C)O2)c(OC)cc1CN(C)C</smiles>

Intermediate 5A

1-(2,5-dimethoxy-4-(4,4,5,5-tetramethyl-1,3,2-dioxaborolan-2-yl)phenyl)-N,N-dimethylmethanamine $[\mathrm{M}+\mathrm{H}]: 322.3, \mathrm{Rt}=0.69 \mathrm{~min}$<smiles>COc1cc(-c2cn(C)c(=O)c3cc(C(F)(F)F)ccc23)c(OC)cc1CN(C)C</smiles>

4-(4-((dimethylamino)methyl)-2,5-dimethoxyphenyl)-2-methyl-7-(trifluoromethyl)isoquinolin-1(2H)-one $3.03 \%, 0.00346 \mathrm{mmol}, 1.6 \mathrm{mg}$

\section{Compound 6}

1H NMR (400 MHz, Chloroform-d) $\delta 8.82-8.75(\mathrm{~m}, 1 \mathrm{H}), 7.96(\mathrm{~d}, \mathrm{~J}=1.5 \mathrm{~Hz}, 1 \mathrm{H}), 7.75$ (dd, J = 8.7, 2.0 $\mathrm{Hz}, 1 \mathrm{H}), 7.51(\mathrm{~s}, 1 \mathrm{H}), 7.36(\mathrm{~d}, \mathrm{~J}=8.5 \mathrm{~Hz}, 1 \mathrm{H}), 7.17(\mathrm{~s}, 1 \mathrm{H}), 6.79(\mathrm{~s}, 1 \mathrm{H}), 3.83(\mathrm{~s}, 3 \mathrm{H}), 3.74(\mathrm{~s}, 3 \mathrm{H}), 3.69$ (s, 3H), $3.66(\mathrm{~s}, 1 \mathrm{H}), 2.42(\mathrm{~s}, 5 \mathrm{H})$. 
$[\mathrm{M}+\mathrm{H}]=421.4, \mathrm{Rt}=0.77 \mathrm{~min}$
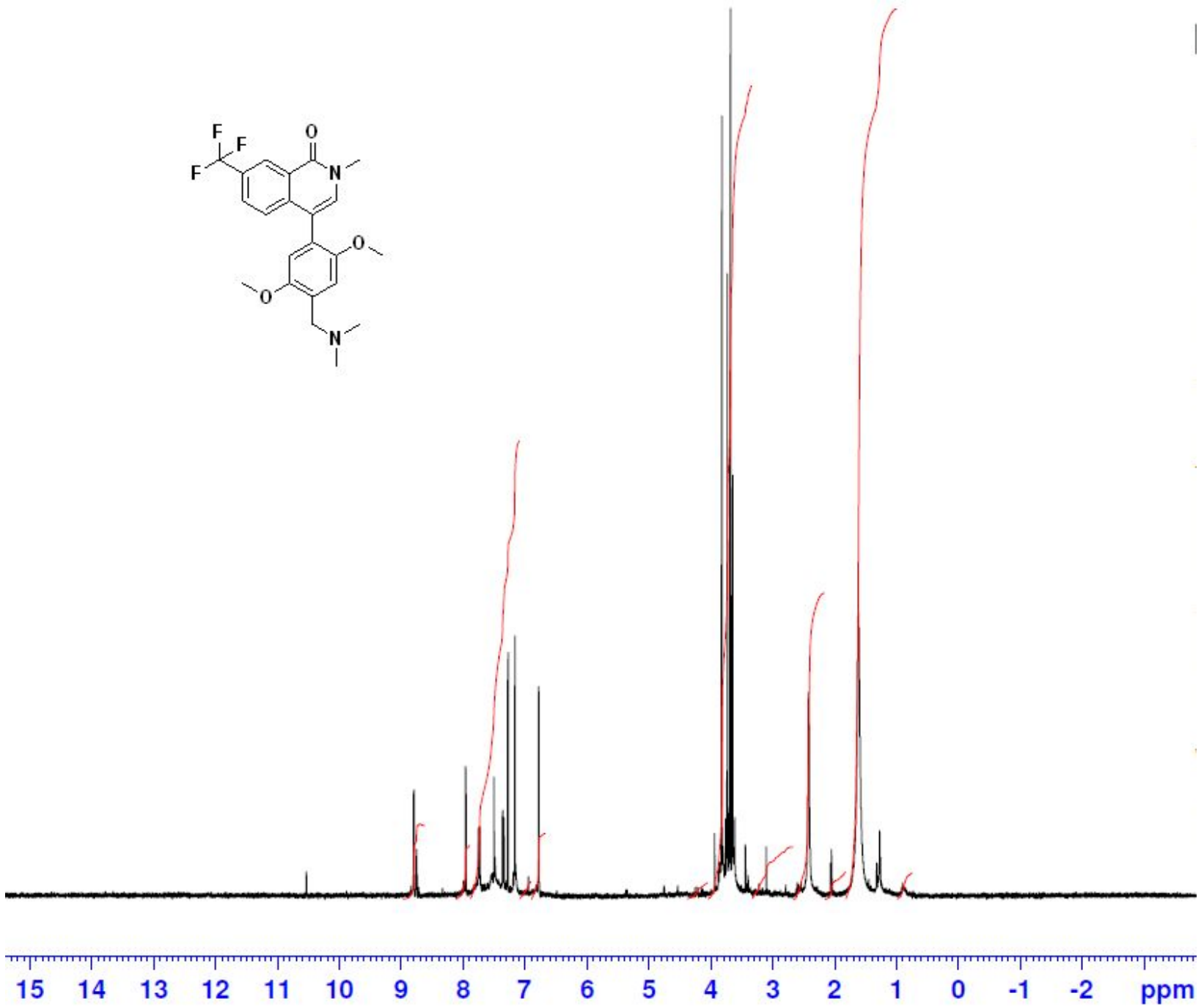<smiles>Cn1ccc2ccc(C(F)(F)F)cc2c1=O</smiles>

\section{Intermediate 6B}

2-methyl-7-(trifluoromethyl)isoquinolin-1(2H)-one

1H NMR (400 MHz, Chloroform-d) $\delta 8.75$ (s, 1H), 7.84 (dd, J = 8.3, 2.0 Hz, 1H), 7.64 (d, J = 8.3 Hz, 1H), $7.21(\mathrm{~d}, \mathrm{~J}=7.3 \mathrm{~Hz}, 1 \mathrm{H}), 6.55(\mathrm{~d}, \mathrm{~J}=7.4 \mathrm{~Hz}, 1 \mathrm{H}), 3.66$ (s, 3H).

$[\mathrm{M}+\mathrm{H}]=229.1$, Rt $0.88 \mathrm{~min}$ 
<smiles>Cn1cc(Br)c2ccc(C(F)(F)F)cc2c1=O</smiles>

Intermediate 6A

4-bromo-2-methyl-7-(trifluoromethyl)isoquinolin-1(2H)-one

$[\mathrm{M}+\mathrm{H}]=308.0, \mathrm{Rt}=1.02 \mathrm{~min}$<smiles>O=c1[nH]ccc2ccc(C(F)(F)F)cc12</smiles>

Commercially available (CAS 410086-28-1); 7-(trifluoromethyl)-1(2H)-isoquinolinone<smiles>CNC(=O)c1ccc2c(-c3cc(OC)c(CN(C)C)cc3OC)cn(C)c(=O)c2c1</smiles>

4-(4-((dimethylamino)methyl)-2,5-dimethoxyphenyl)-N,2-dimethyl-1-oxo-1,2-dihydroisoquinoline-7carboxamide

$8.6 \%, 0.00766 \mathrm{mmol}, 3.3 \mathrm{mg}$

\section{Compound 7}

1H NMR (400 MHz, Methanol-d4) $\delta 8.86(\mathrm{~d}, \mathrm{~J}=1.9 \mathrm{~Hz}, 1 \mathrm{H}), 8.47(\mathrm{~s}, 1 \mathrm{H}), 8.04$ (dt, J = 8.4, $2.5 \mathrm{~Hz}, 1 \mathrm{H}$ ), $7.49-7.43(\mathrm{~m}, 1 \mathrm{H}), 7.31$ (d, J = 8.6 Hz, 1H), 7.23 (d, J = $4.6 \mathrm{~Hz}, 1 \mathrm{H}), 7.13$ (s, 1H), 4.39 (s, 2H), 3.93 (d, J $=1.4 \mathrm{~Hz}, 3 \mathrm{H}), 3.73(\mathrm{~d}, \mathrm{~J}=2.9 \mathrm{~Hz}, 3 \mathrm{H}), 3.70(\mathrm{~s}, 3 \mathrm{H}), 2.98(\mathrm{~s}, 3 \mathrm{H}), 2.92(\mathrm{~s}, 5 \mathrm{H})$

$[\mathrm{M}+\mathrm{H}]=410.4, \mathrm{Rt}=0.86 \mathrm{~min}$ 


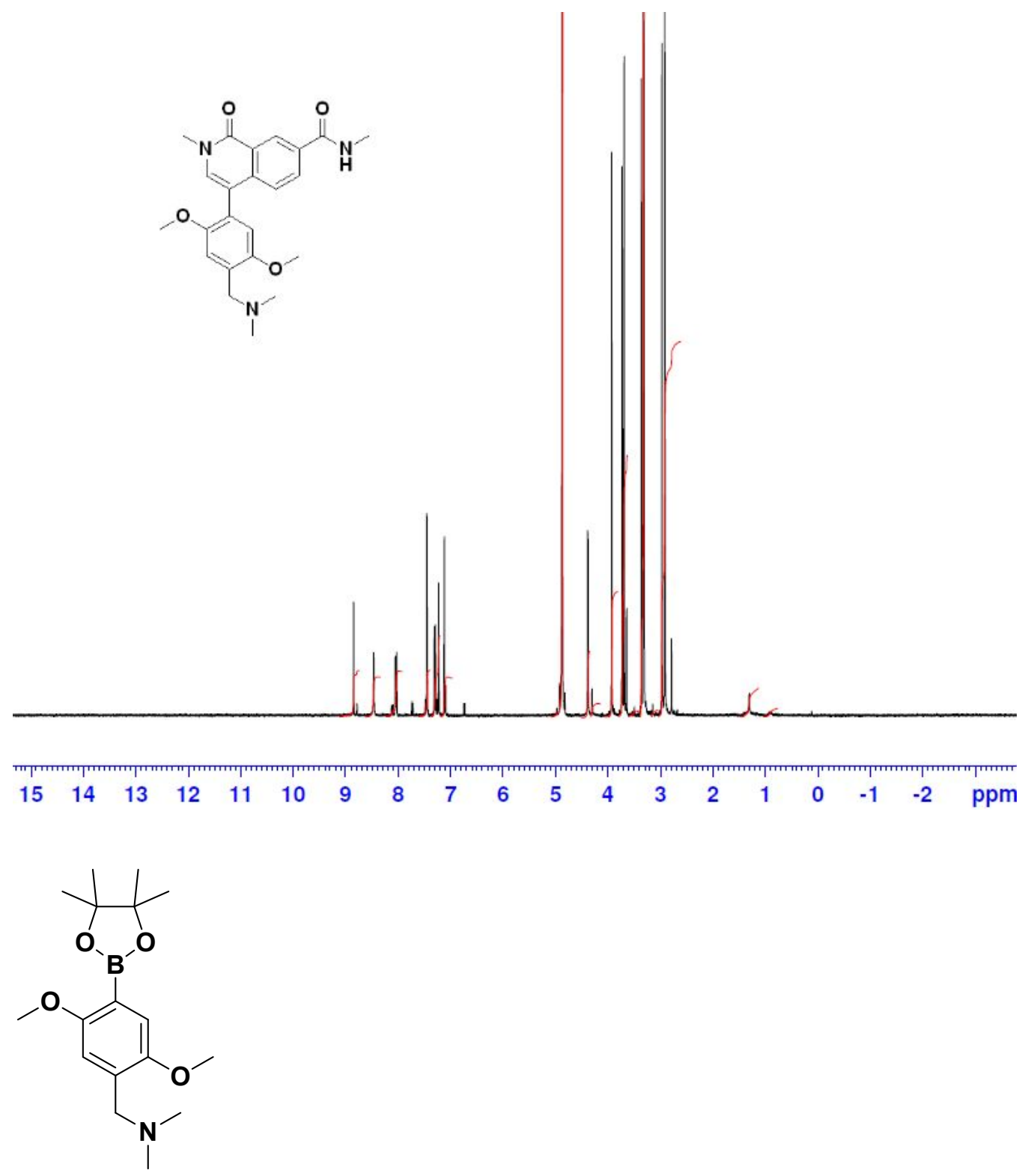

Intermediate 5A

See above<smiles>COC(=O)c1ccc2cc[nH]c(=O)c2c1</smiles>

Commercially available, (CAS 658082-39-4); methyl 1-oxo-12-dihydro-7-isoquinolinecarboxylate 
<smiles>COC(=O)c1ccc2c(Br)c[nH]c(=O)c2c1</smiles>

Intermediate 7A

methyl 4-bromo-1-oxo-1,2-dihydroisoquinoline-7-carboxylate

$[\mathrm{M}+\mathrm{H}]=282.0, \mathrm{Rt}=0.80 \mathrm{~min}$<smiles>COC(=O)c1ccc2c(Br)cn(C)c(=O)c2c1</smiles>

Intermediate 7B

methyl 4-bromo-2-methyl-1-oxo-1,2-dihydroisoquinoline-7-carboxylate $[\mathrm{M}+\mathrm{H}]=298.0, \mathrm{Rt}=0.90 \mathrm{~min}$<smiles>Cn1cc(Br)c2ccc(C(=O)O)cc2c1=O</smiles>

Intermediate 7C

4-bromo-2-methyl-1-oxo-1,2-dihydroisoquinoline-7-carboxylic acid $[\mathrm{M}+\mathrm{H}]=283.9, \mathrm{Rt}=0.72 \mathrm{~min}$<smiles>CNC(=O)c1ccc2c(Br)cn(C)c(=O)c2c1</smiles>

Intermediate 7D

4-bromo-N,2-dimethyl-1-oxo-1,2-dihydroisoquinoline-7-carboxamide $[M+H]=295.0, R t=0.66 \mathrm{~min}$ 


\section{$--$}<smiles>COc1cc(-c2cn(C)c(=O)c3cc(C#N)ccc23)c(OC)cc1CN(C)C</smiles>

4-(4-((dimethylamino)methyl)-2,5-dimethoxyphenyl)-2-methyl-1-oxo-1,2-dihydroisoquinoline-7-carbonitrile $9.9 \%, 0.011 \mathrm{mmol}, 4.5 \mathrm{mg}$

\section{Compound 8}

1H NMR (400 MHz, Chloroform-d) $\delta 8.82(\mathrm{~d}, \mathrm{~J}=1.8 \mathrm{~Hz}, 1 \mathrm{H}), 8.45(\mathrm{~s}, 2 \mathrm{H}), 7.74$ (td, J = 7.9, 7.2, $1.8 \mathrm{~Hz}$, $1 \mathrm{H}), 7.39(\mathrm{~s}, 1 \mathrm{H}), 7.28(\mathrm{~d}, \mathrm{~J}=2.0 \mathrm{~Hz}, 1 \mathrm{H}), 7.20(\mathrm{~s}, 1 \mathrm{H}), 6.83(\mathrm{~s}, 1 \mathrm{H}), 4.23(\mathrm{~d}, \mathrm{~J}=4.4 \mathrm{~Hz}, 2 \mathrm{H}), 3.86(\mathrm{~d}, \mathrm{~J}=$ $9.3 \mathrm{~Hz}, 3 \mathrm{H}), 3.71(\mathrm{~d}, \mathrm{~J}=2.3 \mathrm{~Hz}, 3 \mathrm{H}), 3.69(\mathrm{~s}, 3 \mathrm{H}), 2.76(\mathrm{~s}, 6 \mathrm{H})$.

$[\mathrm{M}+\mathrm{H}]=378.3, \mathrm{Rt}=0.97 \mathrm{~min}$

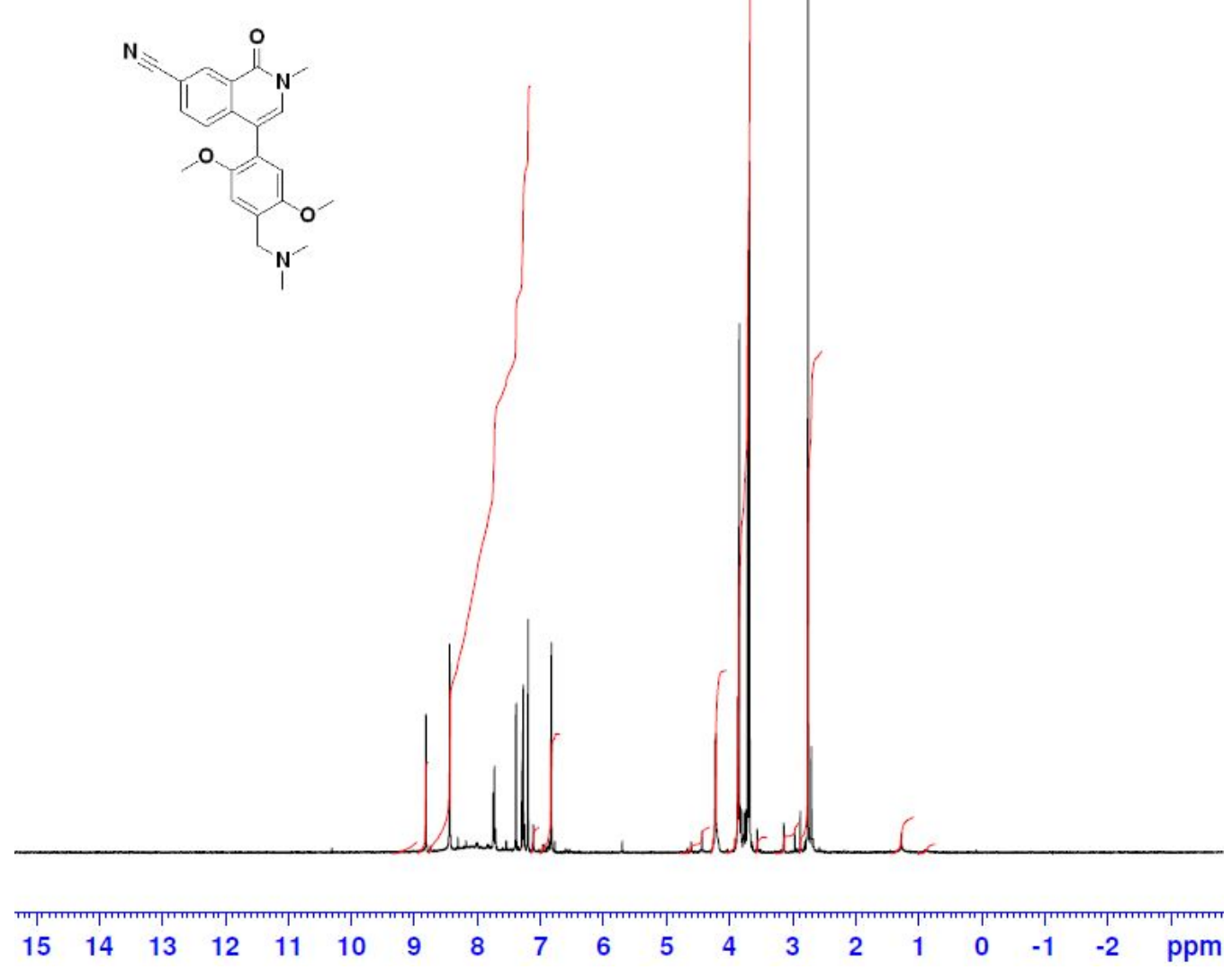




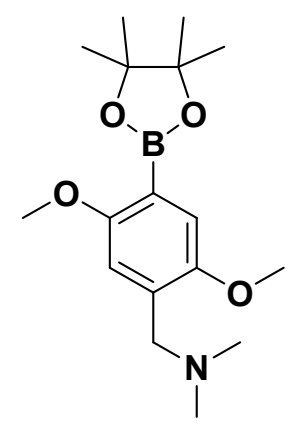

Intermediate 5A

See above<smiles>N#Cc1ccc2cc[nH]c(=O)c2c1</smiles>

Commercially available, (CAS 1184913-64-1); 1-oxo-1,2-dihydroisoquinoline-7-carbonitrile<smiles>Cn1ccc2ccc(C#N)cc2c1=O</smiles>

Intermediate 8A

2-methyl-1-oxo-1,2-dihydroisoquinoline-7-carbonitrile

$[\mathrm{M}+\mathrm{H}]=185.1, \mathrm{Rt}=0.64 \mathrm{~min}$<smiles>Cn1cc(Br)c2ccc(C#N)cc2c1=O</smiles>

\section{Intermediate 8B}

4-bromo-2-methyl-1-oxo-1,2-dihydroisoquinoline-7-carbonitrile

$[\mathrm{M}+\mathrm{H}]=264.9, \mathrm{Rt}=0.81 \mathrm{~min}$

$-$ 
<smiles>COc1cc(-c2cn(C)c(=O)c3cc(F)ccc23)c(OC)cc1CN(C)C</smiles>

4-(4-((dimethylamino)methyl)-2,5-dimethoxyphenyl)-7-fluoro-2-methylisoquinolin-1(2H)-one

$15.2 \%, 0.024 \mathrm{mmol}, 9.5 \mathrm{mg}$

\section{Compound 9}

${ }^{1} \mathrm{H}$ NMR $(400 \mathrm{MHz}$, Chloroform- $d) \delta 8.16(\mathrm{dd}, J=9.4,2.8 \mathrm{~Hz}, 1 \mathrm{H}), 7.35-7.19(\mathrm{~m}, 3 \mathrm{H}), 7.03(\mathrm{~s}, 1 \mathrm{H}), 6.80$ (s, 1H), $3.83(\mathrm{~s}, 3 \mathrm{H}), 3.76(\mathrm{~s}, 5 \mathrm{H}), 3.67(\mathrm{~s}, 3 \mathrm{H}), 2.50(\mathrm{~s}, 6 \mathrm{H})$.

$[\mathrm{M}+\mathrm{H}]=371.3, \mathrm{Rt}=0.95 \mathrm{~min}$
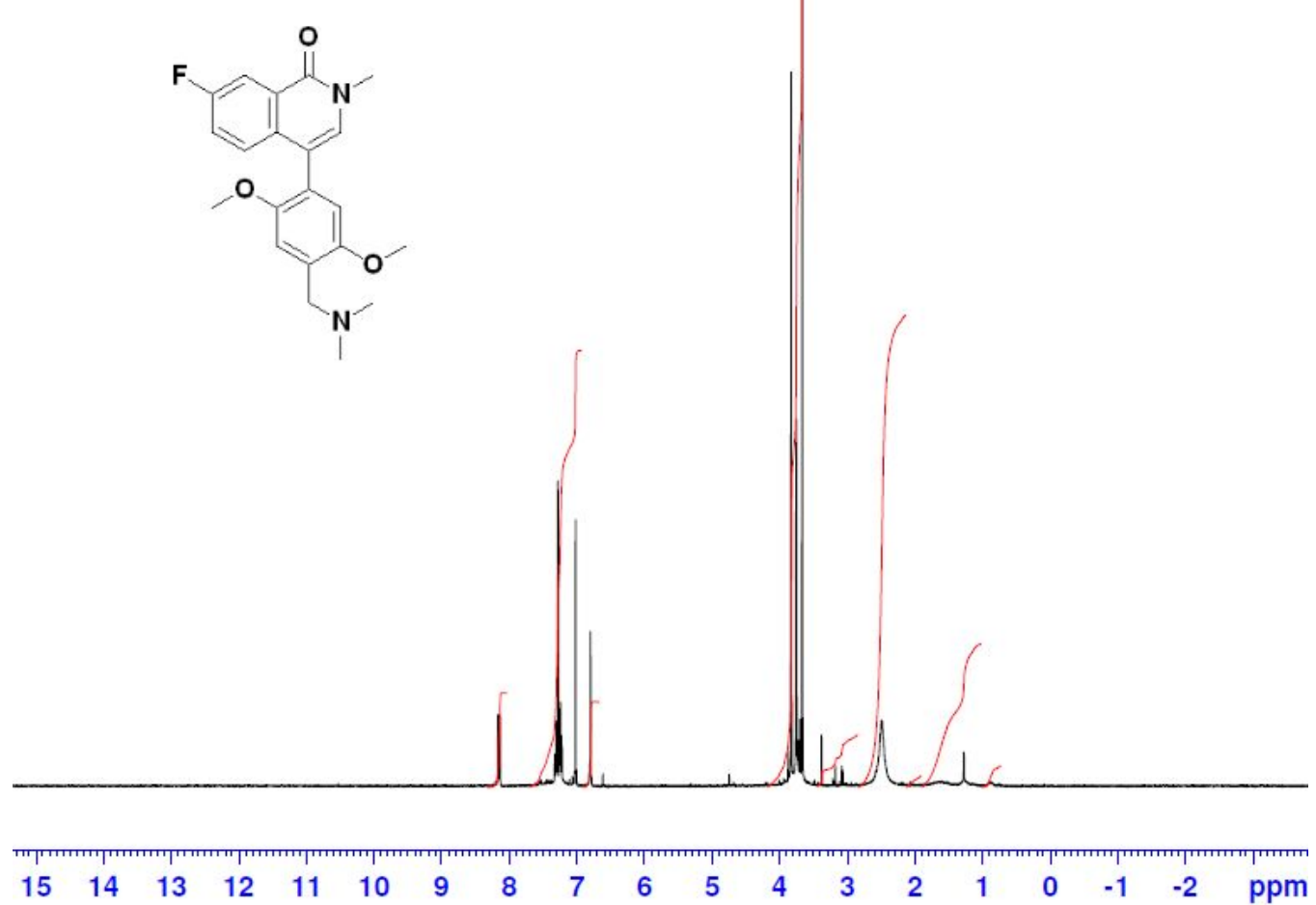


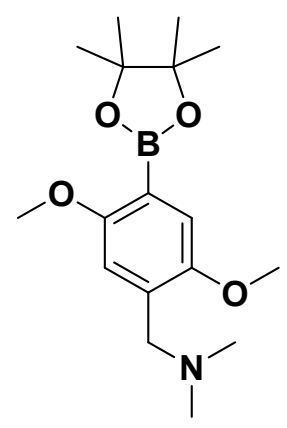

Intermediate 5A

See above<smiles>O=c1[nH]cc(Br)c2ccc(F)cc12</smiles>

Commercially available, (CAS 1227607-99-9); 4-bromo-7-fluoroisoquinolin-1(2H)-one<smiles>Cn1cc(Br)c2ccc(F)cc2c1=O</smiles>

Intermediate 9A

4-bromo-7-fluoro-2-methylisoquinolin-1(2H)-one

$[\mathrm{M}+\mathrm{H}]=255.9, \mathrm{Rt}=0.84 \mathrm{~min}$

$--$

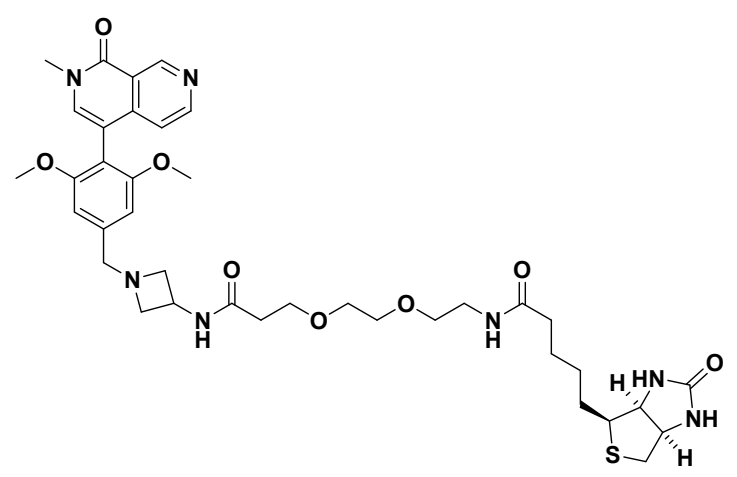

$\mathrm{N}$-(2-(2-(3-((1-(2,5-dimethoxy-4-(2-methyl-1-oxo-1,2-dihydro-2,7-naphthyridin-4-yl)benzyl)azetidin-3yl)amino)-3-oxopropoxy)ethoxy)ethyl)-5-((3aS,4S,6aR)-2-oxohexahydro-1H-thieno[3,4-d]imidazol-4yl)pentanamide 
$24.5 \%, 0.00979 \mathrm{mmol}, 7.5 \mathrm{mg}$

\section{Compound 1 - BIOTIN}

${ }^{1} \mathrm{H}$ NMR $\left(400 \mathrm{MHz}\right.$, Methanol- $\left.d_{4}\right) \delta 9.48(\mathrm{~d}, J=1.0 \mathrm{~Hz}, 1 \mathrm{H}), 8.58(\mathrm{dd}, J=5.8,4.0 \mathrm{~Hz}, 1 \mathrm{H}), 7.61(\mathrm{~s}, 1 \mathrm{H})$, $7.17-7.08(\mathrm{~m}, 2 \mathrm{H}), 6.99(\mathrm{~s}, 1 \mathrm{H}), 5.48(\mathrm{~s}, 4 \mathrm{H}), 4.59-4.42(\mathrm{~m}, 2 \mathrm{H}), 4.28(\mathrm{dd}, J=7.9,4.6 \mathrm{~Hz}, 1 \mathrm{H}), 4.13-$ $3.96(\mathrm{~m}, 4 \mathrm{H}), 3.85(\mathrm{~s}, 2 \mathrm{H}), 3.81-3.70(\mathrm{~m}, 5 \mathrm{H}), 3.67(\mathrm{~d}, J=3.7 \mathrm{~Hz}, 4 \mathrm{H}), 3.60(\mathrm{~d}, J=3.7 \mathrm{~Hz}, 6 \mathrm{H}), 3.52(\mathrm{t}, J$ $=5.5 \mathrm{~Hz}, 3 \mathrm{H}$ ), 2.47 (t, $J=6.1 \mathrm{~Hz}, 2 \mathrm{H}), 1.65$ (tdt, $J=20.8,15.6,7.0 \mathrm{~Hz}, 6 \mathrm{H})$.

$[\mathrm{M}+\mathrm{H}]=766.4, \mathrm{Rt}=0.50 \mathrm{~min}$
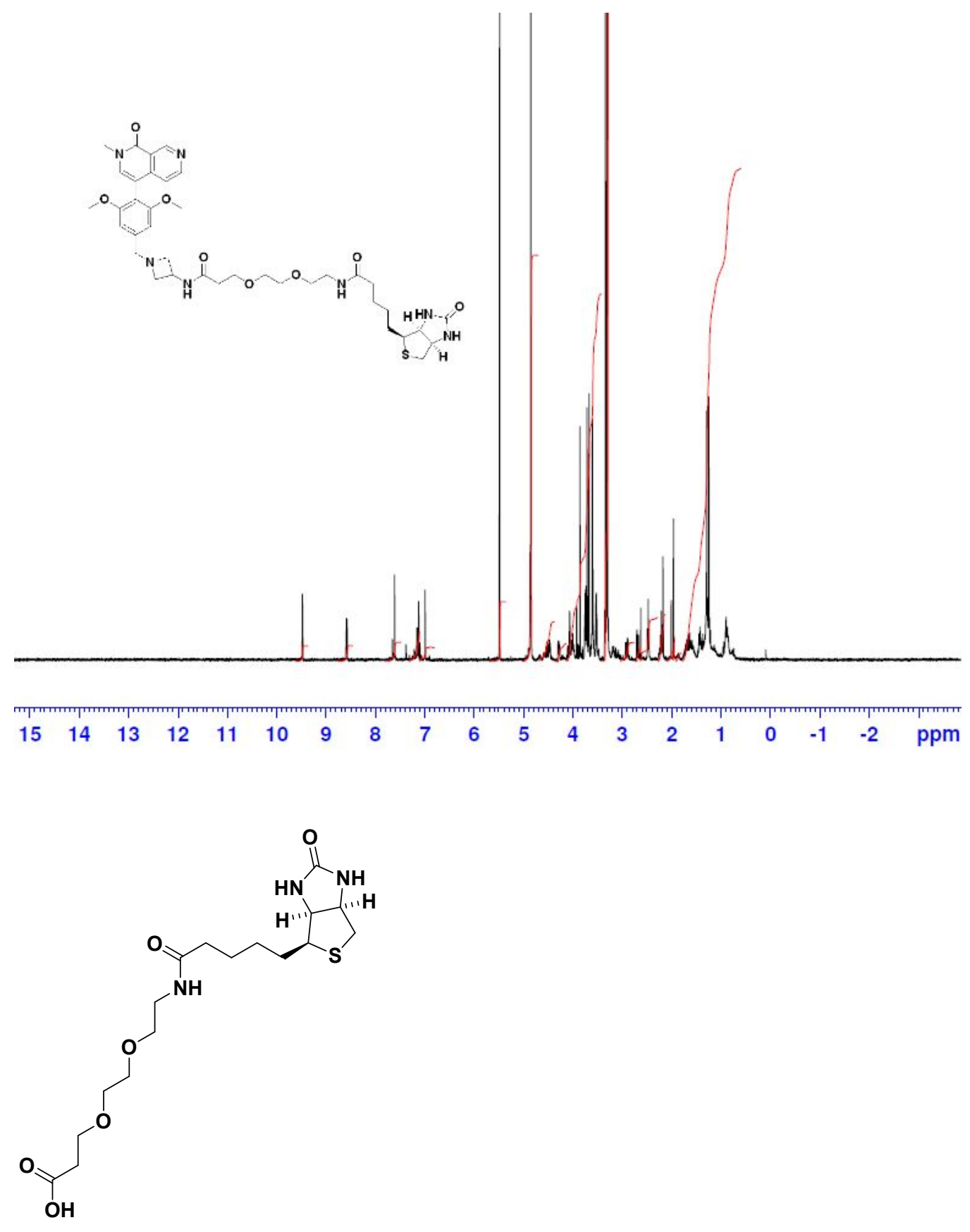

Commercially available, (CAS 1365655-89-5); 3-\{2-[2-(\{5-[(3aS,4S,6aR)-2-oxohexahydro-1H-thieno[3,4d]imidazol-4-yl]pentanoyl\}amino)ethoxy]ethoxy\}propanoic acid 
<smiles>COc1cc(CN2CC(N)C2)cc(OC)c1-c1cn(C)c(=O)c2cnccc12</smiles>

Intermediate 1-BIOTIN-A

4-(4-((3-aminoazetidin-1-yl)methyl)-2,6-dimethoxyphenyl)-2-methyl-2,7-naphthyridin-1(2H)-one $[\mathrm{M}+\mathrm{H}]=381.2, \mathrm{Rt}=1.10 \mathrm{~min}$<smiles>COc1cc(CN2CC(NC(=O)OC(C)(C)C)C2)cc(OC)c1-c1cn(C)c(=O)c2cnccc12</smiles>

Intermediate 1-BIOTIN-B

tert-butyl (1-(3,5-dimethoxy-4-(2-methyl-1-oxo-1,2-dihydro-2,7-naphthyridin-4-yl)benzyl)azetidin-3yl)carbamate

$[\mathrm{M}+\mathrm{H}]=480.9, \mathrm{Rt}=0.59 \mathrm{~min}$<smiles>COc1cc(C=O)cc(OC)c1-c1cn(C)c(=O)c2cnccc12</smiles><smiles>Cn1cc(Br)c2ccncc2c1=O</smiles> 


\section{Intermediates $3 A+1 A$}

See above<smiles>COc1cc(C=O)cc(OC)c1B(O)O</smiles>

Commercially available (CAS 1256355-34-6); 2,6-Dimethoxy-4-forymlphenylboronic acid

$--$<smiles>COc1cc(CN2CC(NC(=O)CCc3ccc(/C=C4/C=CC(c5ccc[nH]5)=N4)n3B(F)F)C2)cc(OC)c1-c1cn(C)c(=O)c2cnccc12</smiles>

(Z)-3-(5-(1H,5'H-[2,2'-bipyrrol]-5'-ylidenemethyl)-1-(difluoroboraneyl)-1H-pyrrol-2-yl)-N-(1-(3,5-dimethoxy4-(2-methyl-1-oxo-1,2-dihydro-2,7-naphthyridin-4-yl)benzyl)azetidin-3-yl)propanamide

$22.3 \%, 0.00261 \mathrm{mmol}, 1.9 \mathrm{mg}$

\section{Compound 3-TRACER}

1H NMR (400 MHz, Chloroform-d) $\delta 9.67$ (s, 1H), 8.59 (d, J = 5.6 Hz, 1H), 7.28 (s, 2H), 7.21 (s, 1H), 7.15 (s, 1H), $7.11-7.07(\mathrm{~m}, 1 \mathrm{H}), 7.02(\mathrm{~d}, \mathrm{~J}=2.4 \mathrm{~Hz}, 2 \mathrm{H}), 6.92(\mathrm{~d}, \mathrm{~J}=4.8 \mathrm{~Hz}, 1 \mathrm{H}), 6.86(\mathrm{~d}, \mathrm{~J}=4.9 \mathrm{~Hz}, 2 \mathrm{H})$, $6.41(\mathrm{~s}, 1 \mathrm{H}), 6.32(\mathrm{~d}, \mathrm{~J}=3.9 \mathrm{~Hz}, 1 \mathrm{H}), 3.73(\mathrm{~s}, 6 \mathrm{H}), 3.65(\mathrm{~s}, 4 \mathrm{H}), 3.37(\mathrm{t}, \mathrm{J}=7.5 \mathrm{~Hz}, 3 \mathrm{H}), 2.74(\mathrm{t}, \mathrm{J}=7.6$ $\mathrm{Hz}, 2 \mathrm{H}), 1.62(\mathrm{~s}, 6 \mathrm{H})$.

$[\mathrm{M}+\mathrm{H}]=692.3, \mathrm{Rt}=0.95 \mathrm{~min}$ 

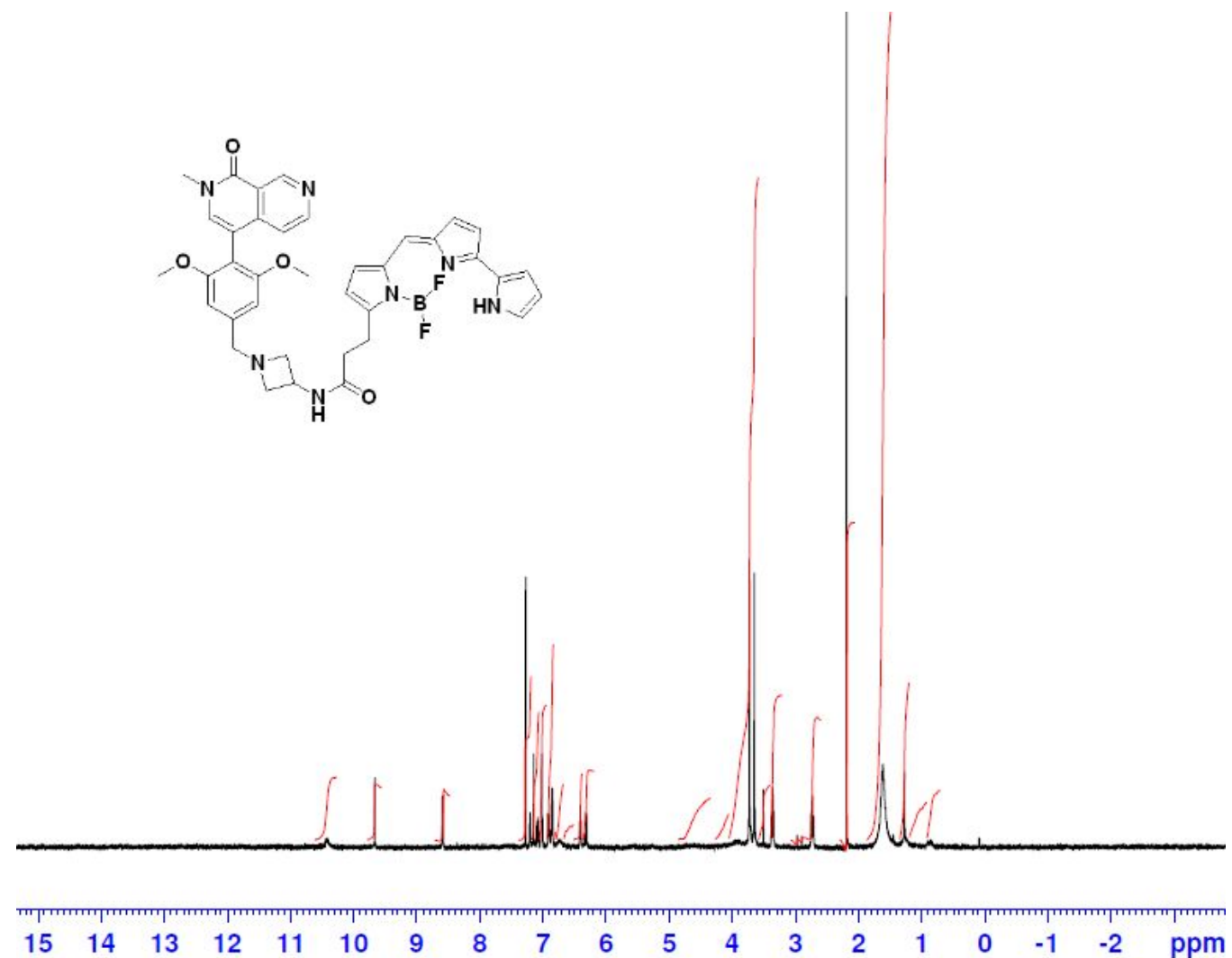<smiles>COc1cc(CN2CC(N)C2)cc(OC)c1-c1cn(C)c(=O)c2cnccc12</smiles>

Intermediate 1-BIOTIN-B

See above 


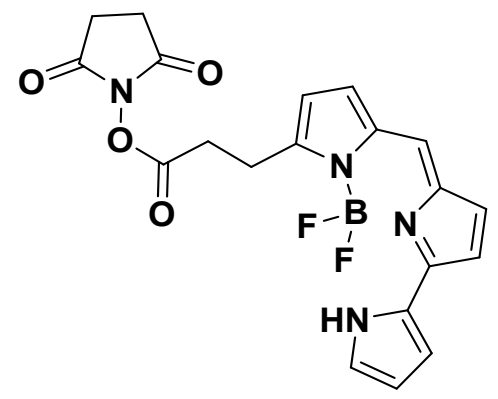

Commercially available, 4,4-difluoro-5-(2-pyrrolyl)-4-bora-3a,4a-diaza-s-indacene-3-propionic acid, succinimidyl ester (BODIPY® 576/589, SE)

$--$<smiles>COc1cc(CN(C)C)cc(OC)c1-c1cn(C)c(=O)c2cc(F)ccc12</smiles>

4-(4-((dimethylamino)methyl)-2,6-dimethoxyphenyl)-7-fluoro-2-methylisoquinolin-1(2H)-one

$15.7 \%, 0.017 \mathrm{mmol}, 6.7 \mathrm{mg}$

\section{Compound DN01}

1H NMR (400 MHz, Chloroform-d) $\delta 8.48$ (s, 1H), 8.14 (dd, J = 9.5, 2.8 Hz, 1H), $7.30-7.22$ (m, 1H), 7.04 $(\mathrm{dd}, \mathrm{J}=8.9,5.2 \mathrm{~Hz}, 1 \mathrm{H}), 6.93(\mathrm{~s}, 1 \mathrm{H}), 6.80(\mathrm{~s}, 2 \mathrm{H}), 3.98(\mathrm{~s}, 2 \mathrm{H}), 3.72(\mathrm{~s}, 6 \mathrm{H}), 3.65(\mathrm{~s}, 3 \mathrm{H}), 2.67(\mathrm{~s}, 6 \mathrm{H})$

$[\mathrm{M}+\mathrm{H}]=371.2, \mathrm{Rt}=0.63 \mathrm{~min}$ 

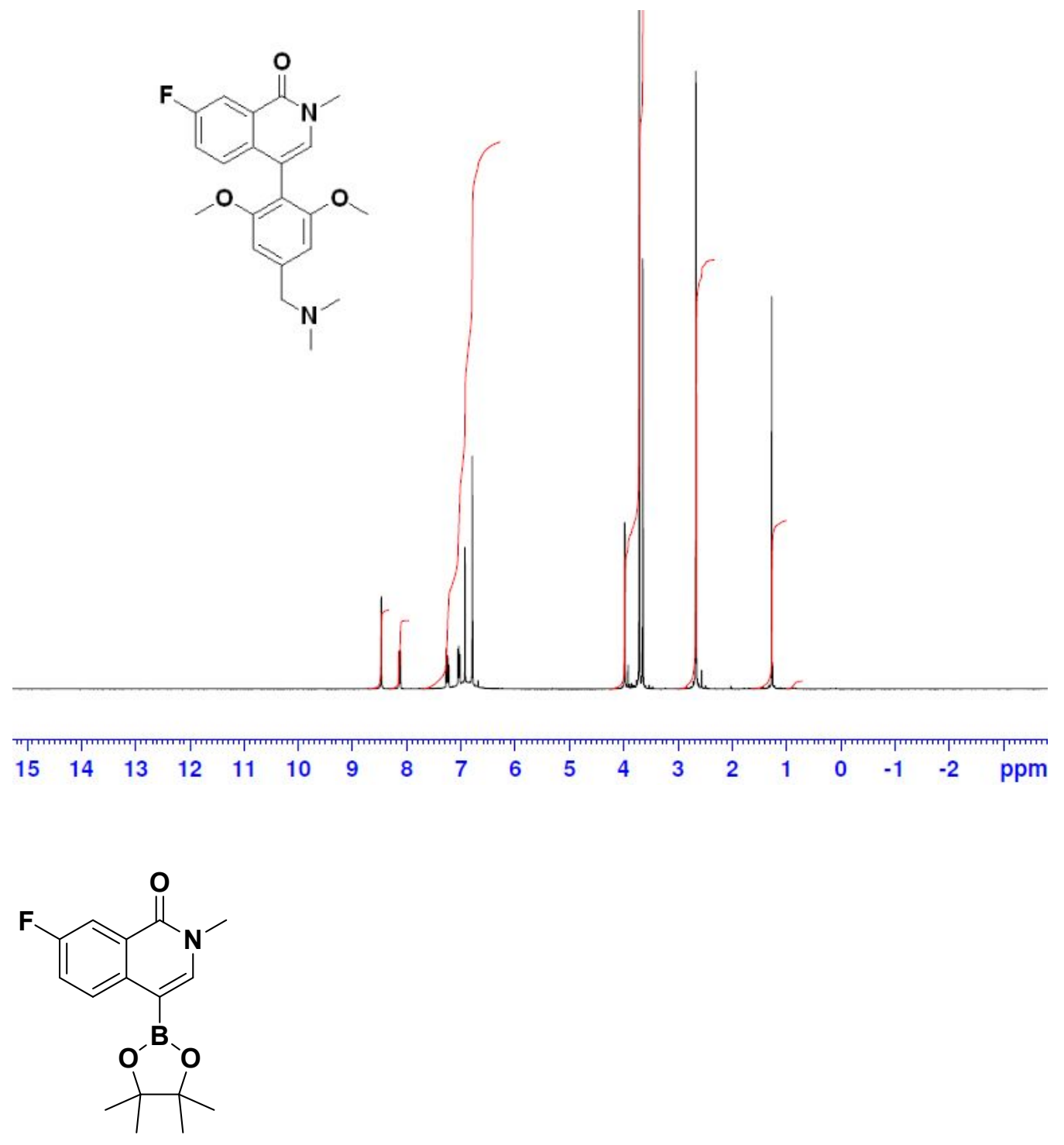

Intermediate DN01-A

7-fluoro-2-methyl-4-(4,4,5,5-tetramethyl-1,3,2-dioxaborolan-2-yl)isoquinolin-1(2H)-one $[\mathrm{M}+\mathrm{H}]=305.2, \mathrm{Rt}=1.14 \mathrm{~min}$<smiles>Cn1cc(Br)c2ccc(F)cc2c1=O</smiles>

Intermediate 9A

See above 
<smiles>COc1cc(CN(C)C)cc(OC)c1Br</smiles>

Intermediate DN01-B

1-(4-bromo-3,5-dimethoxyphenyl)-N,N-dimethylmethanamine

$[\mathrm{M}+\mathrm{H}]=275.1, \mathrm{Rt}=0.54 \mathrm{~min}$<smiles>COc1cc(C=O)cc(OC)c1Br</smiles>

Commercially available, (CAS 31558-40-4); 4-bromo-3,5-dimethoxybenzaldehyde<smiles>COc1cc(CN2CC(N)C2)cc(OC)c1-c1cn(C)c(=O)c2cc(F)ccc12</smiles>

4-(4-((3-aminoazetidin-1-yl)methyl)-2,6-dimethoxyphenyl)-7-fluoro-2-methylisoquinolin-1(2H)-one $34.9 \%, 0.0021 \mathrm{mmol}, 0.9 \mathrm{mg}$

\section{Compound DN02}

1H NMR (400 MHz, Chloroform-d) $\delta 8.14$ (dd, J = 9.5, $2.7 \mathrm{~Hz}, 1 \mathrm{H}), 7.24$ (td, J = 8.5, $2.8 \mathrm{~Hz}, 1 \mathrm{H}$ ), 7.07 (dd, $\mathrm{J}=8.9,5.2 \mathrm{~Hz}, 1 \mathrm{H}), 6.93(\mathrm{~s}, 1 \mathrm{H}), 6.69(\mathrm{~s}, 2 \mathrm{H}), 3.80(\mathrm{~s}, 4 \mathrm{H}), 3.73(\mathrm{~s}, 7 \mathrm{H}), 3.65(\mathrm{~s}, 3 \mathrm{H})$.

$[\mathrm{M}+\mathrm{H}]=398.2, \mathrm{Rt}=0.70 \mathrm{~min}$ 

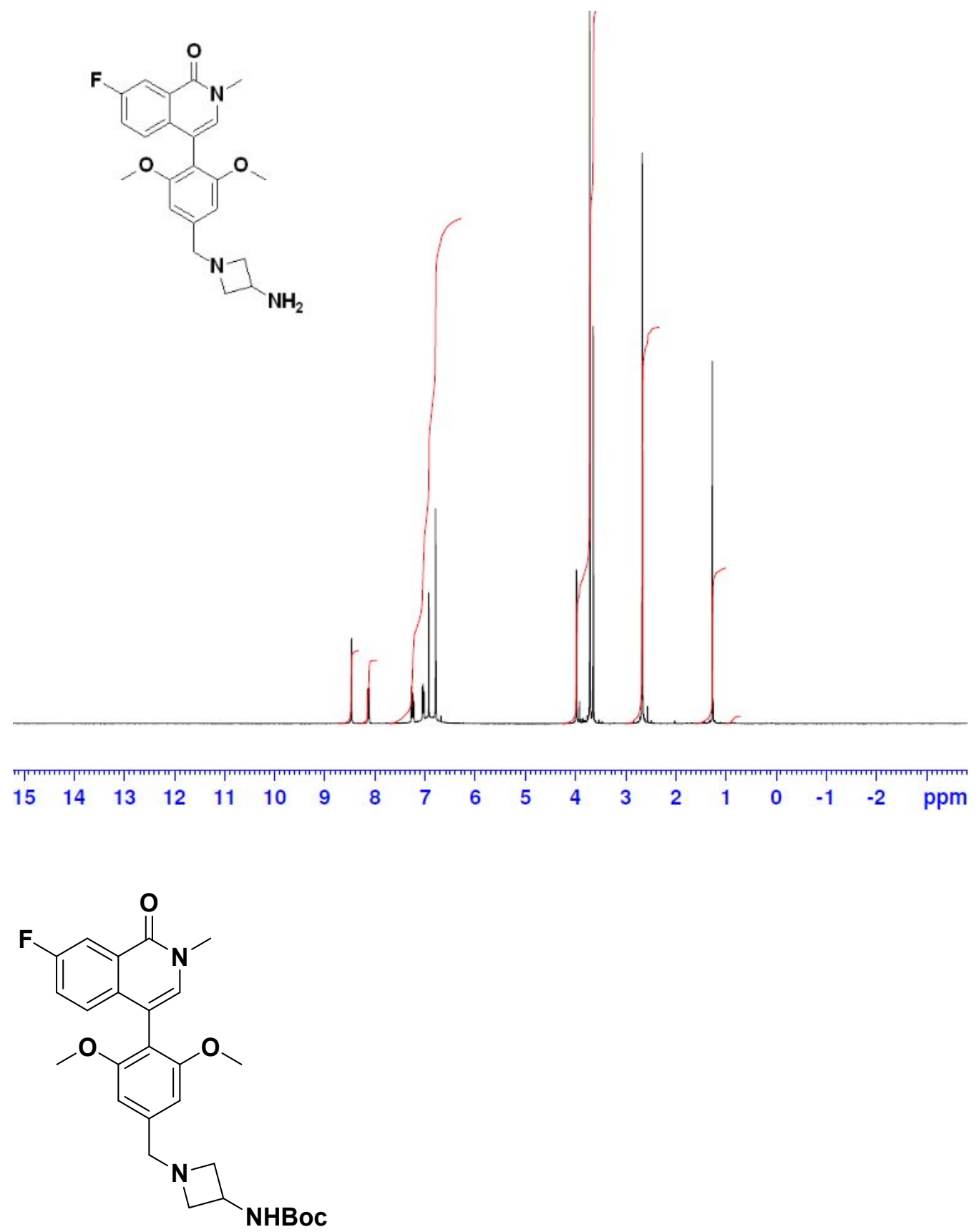

\section{Intermediate DN02-B}

tert-butyl (1-(4-(7-fluoro-2-methyl-1-oxo-1,2-dihydroisoquinolin-4-yl)-3,5-dimethoxybenzyl)azetidin-3yl)carbamate

$[\mathrm{M}+\mathrm{H}]=498.5, \mathrm{Rt}=0.78 \mathrm{~min}$ 


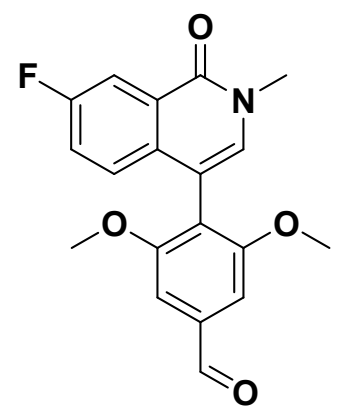

Intermediate DN02-A

4-(7-fluoro-2-methyl-1-oxo-1,2-dihydroisoquinolin-4-yl)-3,5-dimethoxybenzaldehyde $[\mathrm{M}+\mathrm{H}]=342.1, \mathrm{Rt}=0.88 \mathrm{~min}$

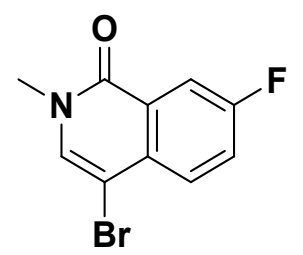

Intermediate 9A

See above<smiles>COc1cc(C=O)cc(OC)c1B(O)O</smiles>

Commercially available (CAS 1256355-34-6); 2,6-Dimethoxy-4-forymlphenylboronic acid 